\title{
Special issue on the 5th international conference on optimization and control with applications (OCA5)
}

\author{
Honglei Xu • Kok Lay Teo • Xinmin Yang • \\ Mingyong Lai
}

Published online: 10 November 2013

(C) Springer-Verlag Berlin Heidelberg 2013

The 5th International Conference on Optimization and Control with Applications (OCA5) was held at the China University of Petroleum, Beijing, China on December 4-8, 2012. As a continuation of the OCA series, OCA5 provided an international forum for scientists, engineers, researchers and practitioners to exchange ideas and approaches, to present research findings and state-of-the-art solutions, to share experiences on potentials and limits and to open new avenues of research and developments on all issues and topics related to optimization and control. More than 200 representatives from over 20 countries and regions such as China, United States, Canada, Australia, Russia and Japan presented their latest work and discussed new progresses on optimization and control with applications. Conference participants were then invited to submit their expanded papers to Optimization Letters (OPTL).

After peer reviews of all the submitted papers in accordance with the usual stringent reviewing process of OPTL, ten (10) papers have been accepted for publication in this special issue. The topics covered in this special issue include: derivative variation approaches for optimal control, primal-dual interior point methods for optimal design, penalty methods for finite-dimensional obstacle problem, modified

\footnotetext{
H. Xu · K. L. Teo $(\varangle)$

Department of Mathematics and Statistics, Curtin University, Bentley, WA, Australia e-mail: K.L.Teo@curtin.edu.au

H. Xu

e-mail: H.Xu@curtin.edu.au

X. Yang

Department of Mathematics, Chongqing Normal University, Chongqing 400047, China e-mail: xmyang@cqnu.edu.cn

M. Lai

School of Economics and Trade, Hunan University, Changsha, Hunan 410079, China e-mail: laimingyong0731@ hotmail.com
} 
nonmonotone BFGS algorithms for smooth nonlinear equations, unified optimization of $H_{\infty}$ and upper stability bound for singularly perturbed systems, stability analysis and optimal control of stochastic singular systems, stability for differential mixed variational inequalities, minimization of the difference of nonconvex vector-valued mappings, set-valued optimization problems in real ordered linear spaces and subdifferentials of perturbed minimal time function in normed space. We hope that this special issue will be a source of valuable references for researchers and practitioners in optimization and optimal control community.

It is with great pleasure and honor for us to have this opportunity to organize the special issue for OPTL. We would like to express our sincerest thanks to Professor Panos M. Pardalos for his valuable advice and encouragement. We also wish to thank Professor Oleg Prokopyev and Professor Pavlo Krokhmal for their follow-up support and encouragement. We thank and appreciate all the authors for their contributions and all the reviewers for their time and effort. We thank the support from the Australian Research Council and the National Natural Science Foundation of China (No. 11171019, No. 61290325). We are grateful to Mrs. Lisa Holling for her editorial assistance. Thanks are also due to the conference participants, committee members and conference sponsors for their tremendous support. 